\title{
pQuattro vectors allow one-step multigene metabolic engineering and auto-selection of quattrocistronic artificial mammalian operons
}

Short Communication

\author{
Martin Fussenegger, Samuel Moser \& James E. Bailey* \\ Institute of Biotechnology, Swiss Federal Institute of Technology, ETH Zurich, CH-8093 Zurich, Switzerland
}

Received 25 August 1998; accepted 25 August 1998

Key words: CITE, EMCV, GFP, IRES, picornavirus

\begin{abstract}
Based on internal ribosomal entry sites (IRES) of picornaviral origin we constructed a novel family of mammalian expression vectors. pQuattro vectors contain quattrocistronic artificial eukaryotic operons which link, in a single transcript, the simultaneous and coordinated as well as adjustable expression of up to three independent genes of interest to a terminal neomycin (neo) resistance marker. Due to the strict genetic linkage of the transgenes and the terminal selection marker, this genetic configuration enables, by the selection on neomycin, multigene metabolic engineering of mammalian cells in a single step (one-step metabolic engineering). Furthermore, selection on the terminal cistron of multicistronic expression units enforces cocistronic expression of all upstream encoded genes and maximises genetic integrity of the eukaryotic operon in stable mammalian cell lines, since clones harbouring damaged multicistronic expression units become neomycin-sensitive and are automatically counterselected (autoselection). The modular set-up and the abundance of restriction sites in pQuattro vectors facilitate the movement of individual genes between multicistronic expression vectors and guarantees high compatibility with genetic elements of a wide variety of existing mammalian expression vectors.
\end{abstract}

\section{Introduction}

Current metabolic engineering technology is in most cases based on the transfection of a single transgene, the expression of which is maintained and selected for by cotransfection of a second vector encoding a selectable marker which often confers resistance to an antibiotic. However, due to unlinked expression of the selection marker and the gene of interest, rarely more than $30 \%$ of the stable integrants expressing the antibiotic resistance also express the desired transgene (Fussenegger et al., 1998a). This phenomenon either results from a deletion or inactivation of the gene of interest during or following plasmid integration or from the exclusive integration of the vector encoding the resistance marker. Even if stable cell lines with concomitant expression of the selection marker and the gene of interest could be generated, the expression level of the transgene is unpredictable

* Author for all correspondence. and often decreases during extended cultivations since there is no positive selection for the maintenance and the transcriptional activity of the transgene.

Furthermore, as contemporary basic research continues to reveal more and more details on the molecular complexity of metabolic pathways and networks, classical one-gene metabolic engineering is becoming increasingly limited in its potential to realised desired global phenotypes. In order for metabolic engineering to keep up with the current detailed knowledge of physiological pathways and metabolic networks simultaneous expression of multiple genes is a prerequisite to achieve difficult-to-attain metabolic characteristics or cell culture states (Papoutsakis, 1998; Fussenegger et al., 1998b). Although current expression technology may in principle enable such multigene metabolic engineering by subsequent rounds of transfections with a single gene, such methodology is tedious, time consuming, and increases the risk of generating secondary effects unrelated to the transgenes 
at every round of transfection due to random integrations of the different vectors into the host chromosome (Fussenegger et al., 1998a).

\section{Results and discussion}

Based on specific viral genetic elements which enable cap-independent translation initiation (internal ribosomal entry sites, IRES; Fussenegger et al., this issue for a review), multicistronic expression technology was recently established and successfully used for multigene metabolic engineering of Chinese hamster ovary cells (CHO) (Papoutsakis, 1998; Fussenegger et al., 1998b; Fussenegger et al., 1998c). The concept of multicistronic eukaryotic expression leading to the quattrocistronic family of mammalian expression vectors is based on the previously reported pTRIDENT system (Fussenegger et al., 1998d). The tricistronic expression unit contained in plasmid pTRIDENT1 consists of a tetracyclinerepressible promoter $\left(\mathrm{P}_{h C M V *-1}\right)$ and three multiple cloning sites which are separated by two identical IRES elements of polioviral origin (Fussenegger et al., 1998d). In order to extend this tricistronic expression unit to the quattrocistronic level by the addition of a selectable marker (pQuattro1), the SspI/BglII fragment of pTRIDENT1 $\left(\mathrm{P}_{h C M V *-1}\right.$-MCSI-IRESIMCSII-IRESII-MCSIII) was fused to the corresponding fragment of pIRESneo (SspI/BglII; Clontech, Palo Alto, CA; Rees et al., 1996) containing a capindependent translation enhancer (CITE) an IRES element of the encephalomyocarditis virus (EMCV) fused to the neomycin resistance gene as well as the pUC-derived origin of replication (ori) for high copy number amplification in E. coli (Figure 1; Table 1). Additional quattrocistronic vectors pQuattro2, pQuattro3 and pQuattro4 containing different regulatable as well as constitutive promoters such as the ecdysone-responsive promoter $\left(\mathrm{P}_{E C} ; \mathrm{pQuattro} 2\right)$, the SV40 promoter (pQuattro3) and the MPSV promoter (pQuattro4) were constructed by replacing $\mathrm{P}_{h C M V *-1}$ of pQuattro1 by the respective promoter elements (SspI/EcoRI) of pIND (Invitrogen), pSBC1 (Dirks et al., 1993), and pMPSVEH (Dirks et al., 1994), respectively (Figure 1).

In order to test the quattrocistronic expression unit, pQuattro3 and pQuattro4 derivatives pMF143 and pMF144 were constructed which harbour the secreted alkaline phosphatase (seap, Berger et al., 1988), the tumor suppressor gene p21 (El-Deiry et al., 1993), the green fluorescent protein (GFP; Crameri et al., 1996) and the neomycin resistance gene (Rees et al., 1996; Table 1). In addition, a pQuattro1 derivative was constructed to assess tetracycline-dependent regulation of the multicistronic expression unit (data not shown; Fussenegger et al., 1998d). pMF143 and pMF144 resulted from an EcoRI/BglII fusion of the pTRIDENT1 derivative pMF132 (Fussenegger et al., 1998d) and the respective pQuattro vectors (Table 1). pMF143 and pMF144 were transfected into wildtype CHO-K1 cells (ATCC: CCL61) as described before (Fussenegger et al., 1997a). Transfected populations were grown in FMX-8 medium (Messi Cell Culture Technology, Switzerland) supplemented with $10 \%$ fetal calf serum (FCS, Boehringer Mannheim, Lot Nr. 14713602) and for the selection of stable transfectants also with $400 \mu \mathrm{L} \mathrm{mL}^{-1} \mathrm{G} 418$. The neomycin-resistant cell population was qualitatively tested for expression of SEAP, p21, and GFP using an enzymatic assay or (immuno)fluorescence microscopy (Berger et al., 1988; Fussenegger et al., 1997a, Figure 2). Besides the SEAP activity measured in the cell culture supernatant the transfected cells show a coordinated expression of p21 and GFP (Figure 2). Owing to the cytostatic effect of the tumor suppressor gene p21, transfected cells stop their cell cycle and cell division following transfection of the quattrocistronic expression vector. Consequently, cells harbouring the pQuattro derivatives pMF143 and pMF144 are not able to proliferate and remain single for extended time periods of at least 4 weeks and probably beyond that period (Figure 2). Because of the instantaneous proliferation arrest of CHO cells transfected with pMF143 and pMF144, SEAP, p21 and GFP expression levels were assessed in transient transfection experiments. The SEAP production derived from pMF143 and pMF144 was identical to levels expressed from pTRIDENT derivatives or from isogenic mono- or dicistronic expression vectors (Fussenegger et al., 1997a; Fussenegger et al., 1998d). Similarly, (immuno)fluorescence intensities of p21 and GFP were comparable to the ones derived from isogenic di- or tricistronic configurations (Fussenegger et al., 1997a; Fussenegger et al., 1998d).

Despite this complete proliferation block the cells display no morphologies suggestive of apoptosis and seem to remain viable since their cell membranes retain intracellular GFP during the entire time period (Figure 2; Fussenegger et al., 1997a). Certainly, p21 has been previously shown to arrest $\mathrm{CHO}$ cells at G1phase in transient transfection experiments at high intracellular concentrations (Fussenegger et al., 1997a) 

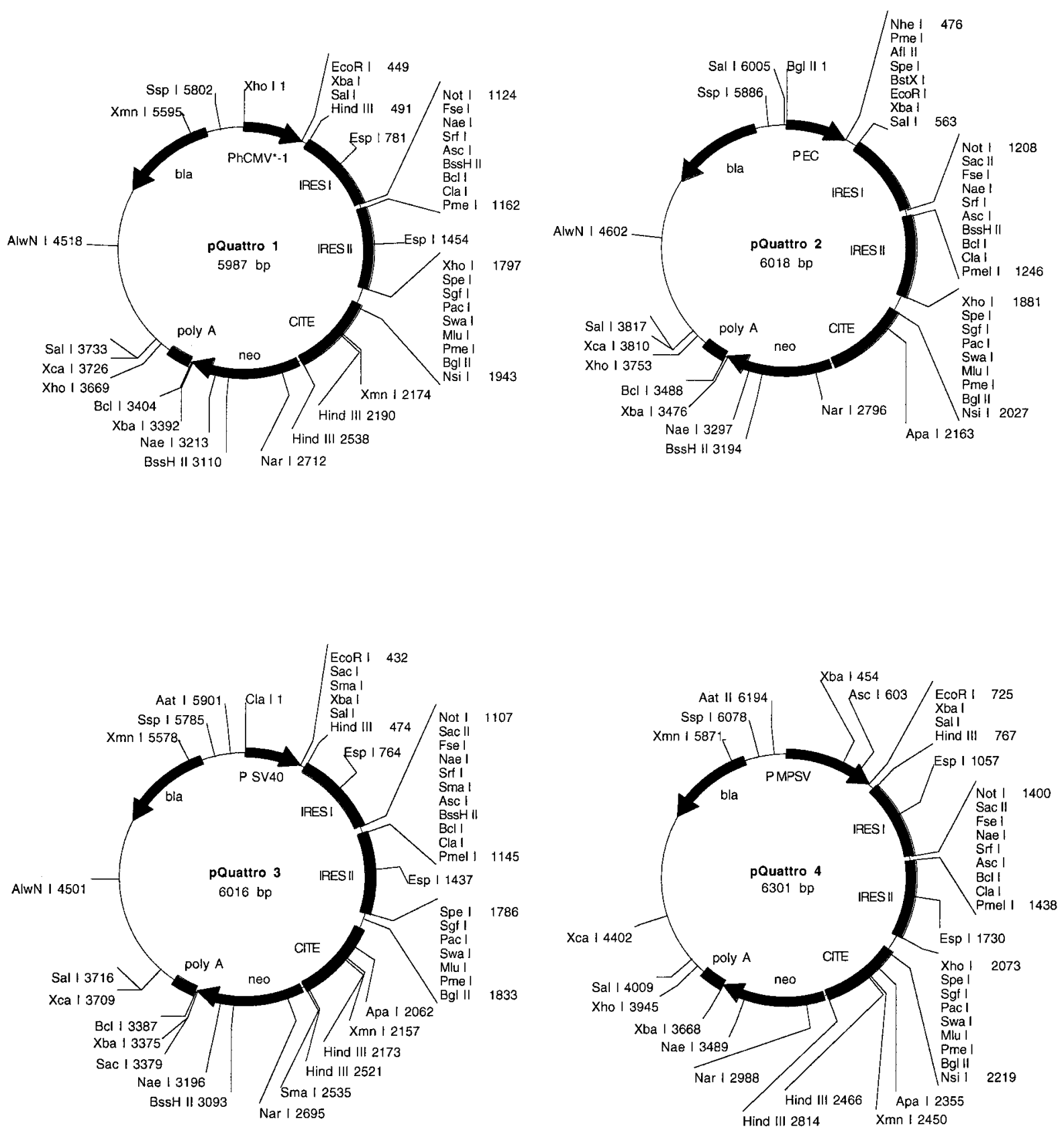

Figure 1. Maps of pQuattro1-4. All mammalian expression vectors contain the high copy number pUC-derived ori as well as the ampicillin resistance gene (bla) for high level amplification and selection in $E$. coli. The quattrocistronic expression units consist of three multiple cloning sites, eukaryotic promoter elements $\mathrm{P}_{h C M V *-1}$ (tetracycline-responsive), $\mathrm{P}_{E C}$ (ecdysone-responsive) or the constitutive viral promoters $\mathrm{P}_{S V 40}$ and $\mathrm{P}_{M P S V}$, two internal ribosomal entry sites (IRES), a cap-independent translation enhancer (CITE) fused to the G418 resistance gene $(n e o)$, and a terminal polyadenylation signal $(p A)$ derived from bovine growth hormone. 

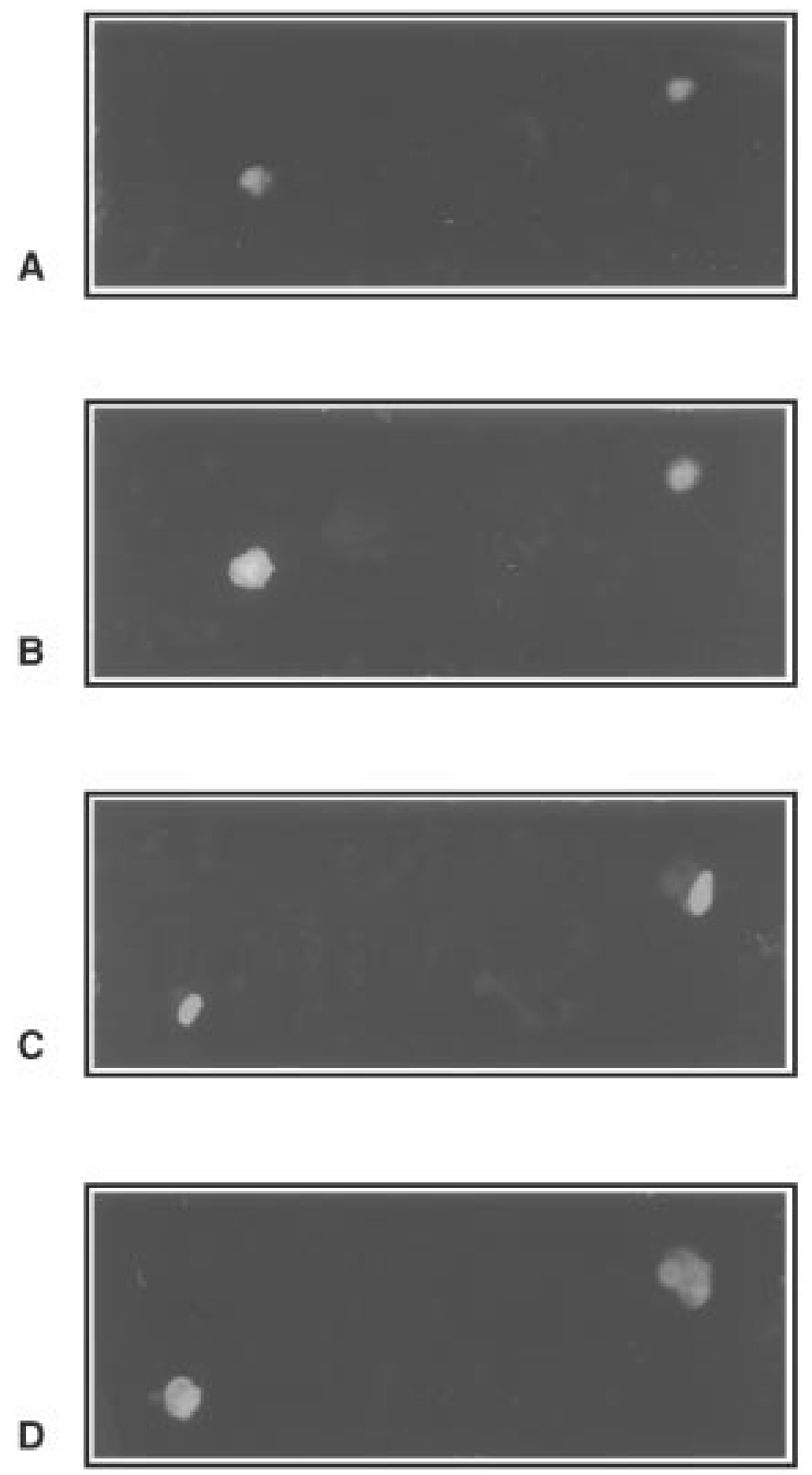

Figure 2. Immunofluorescence of CHO-K1 cells stably transfected with pMF143 (A and B) and pMF144 (C and D). A and C show immunofluorescence of p21 encoded by the second cistron whereas B and D show GFP-mediated green fluorescence. The expression of both genes is strictly correlated due to their expression from a single expression unit. 
Table 1. Plasmids used in this study

\begin{tabular}{lll}
\hline Plasmid & Description & Reference or source \\
\hline pIND & Cloning vector containing the ecdysone-regulatable promoter $P_{E C}$ & Invitrogen \\
pIRESneo & Dicistronic expression vector encoding neomycin resistance in the second cistron & Clontech/Rees et al., 1996 \\
pMPSVEH & Cloning vector containing the strong, constitutive viral promoter $\mathrm{P}_{M P S V}$ & Dirks et al., 1994 \\
pSBC1 & Cloning vector containing the strong, constitutive viral promoter $\mathrm{P}_{S V 40}$ & Dirks et al., 1993 \\
pTRIDENT1 & Tricistronic mammalian expression vector & Fussenegger et al., 1997b \\
pMF132 & pTRIDENT1-derivative harboring seap-p21-gfp in a tricistronic expression unit & Fussenegger et al., 1997b \\
pMF143 & pQuattro3 containing the tricistronic seap-p21-gfp-containing expression unit & This work \\
& of pMF132 (EcoRI/BglII) & This work \\
pMF144 & pQuattro4 containing the trisictronic seap-p21-gfp-containing expression unit & This work \\
pQuattro1 & of pMF132 (EcoRI/BglII) & This work \\
pQuattro2 & pQlII/SspI fusion of pTRIDENT1 and pIRESneo & This work \\
pQuattro3 & pQuattro1 containing the SV40 promoter of pSBC1 (EcoRI/SspI) & This work \\
pQuattro4 & pQuattro1 containing the MPSV promoter of pMPSVEH $(E c o R I / S s p I)$ & \\
\hline
\end{tabular}

but stable transfection of regulated, $\mathrm{P}_{h C M V *-1}$-based p21-expression vectors such as recently developed for the cytostatic production process were not able to arrest the cell cycle, most probably due to insufficient expression levels (Fussenegger et al., 1997a; Fussenegger et al., 1998c).

The pQuattro family of mammalian expression vectors is a highly modular multicistronic expression system which allows the simultaneous, coordinated and, in some cases, adjustable expression of up to three genes of interest which are transcriptionally linked to the neomycin resistance marker in the terminal cistron. The concept of the quattrocistronic expression vectors presented here has several advantages over existing mammalian expression systems and has been designed for maximum compatibility with existing and future expression technologies:

(1) pQuattro vectors contain three multiple cloning sites harbouring up to 19 unique restriction sites (pQuattro2), 7 of which represent 8 bp target sequences for restriction endonucleases. The abundance of unique restriction sites present combined with the modular configuration of the functional genetic elements (promoter cassettes, IRES and CITE elements) avoids conflicting cloning situations arising from consecutive gene integrations, guarantees optimal compatibility with existing di- and tricistronic mammalian expression vectors (Dirks et al., 1993; Dirks et al., 1994; Rees et al., 1996; Fussenegger et al., 1998d) and allows the downsizing of pQuattro to tri- and dicistronic vectors by deleting individual IRES elements using either restriction sites contained in the MCS (PmeI, BclI/BglII; SrfI/SwaI; BssHII/MluI) or in the IRES elements (EspI). The vector backbone as well as the neomycin resistance cassette are most easily replaced using a restriction site of the various MCS and the SspI (or XmnI) sites contained in the promoter region of the ampicillin resistance gene (bla). Also, the pQuattro vectors are compatible to the pIRESpuro (Clontech) vector for $S s p \mathrm{I} / B g l$ II replacement of the neomycin cassette with the puromycin resistance gene and to the dual selection marker vectors pNeoEGFP and pHygEGFP (Clontech) via $S s p \mathrm{I} / M l u \mathrm{I}$ sites. Furthermore, the promoter cassette of pQuattro vectors can be replaced by corresponding $S s p \mathrm{I} / E c o$ RI promoter elements contained in a great variety of existing mammalian expression vectors (Dirks et al., 1993 and 1994; Fussenegger et al., 1998). The modular structure of the pQuattro vector family allows straightforward adaptation for special applications and future expression technologies. Additionally, useful promoter-IRESI-IRESII, IRESI-IRESII, IRESI-IRESIIneo cartridges can be generated from the pQuattro vectors.

A single AatII site at the $5^{\prime}$ end of the multicistronic expression unit which is present in all pQuattro vectors and allows further modifications and integration of additional genetic elements.

(2) The pQuattro family of multicistronic expression vectors allows the simultaneous and coordinated expression of up to three genes of interest which is most useful for (i) multigene engineering of com- 
plex metabolic pathways (e.g. cell cycle and apoptosis or glycosylation; Fussenegger et al., submitted), (ii) evaluation of multiple gene functions (functional genomics), (iii) tissue engineering as well as cell and gene therapy, (iv) auto-regulated expression systems (Fussenegger et al., 1997b), (v) production of multiprotein complexes (e.g. antibodies), (vi) production of viral packaging cell lines (expression of gag, env, pol), (vii) multi-antigen/epitope (DNA) vaccination or (viii) the combination of antigens with immunomodulatory or -stimulatory components (adjuvants). Furthermore, the use of developmentally regulated IRES elements (Bernstein et al., 1997) in multicistronic expression units could superimpose additional translational regulation on multigene metabolic engineering approaches which would be especially valuable for technologies such as tissue engineering and gene therapy.

(3) pQuattro allows novel polycistronic 'one-step auto-select' expression technology. Since the selection marker is encoded by the terminal cistron and transcriptionally and translationally linked to the multicistronic expression unit (one-step multigene metabolic engineering), selection on neomycin ensures the presence of all 5' cistrons ('auto-select' engineering) and exerts a positive selection pressure for the maintenance of all genes encoded on the multicistronic expression unit in an intact and transcriptional active chromosomal context. Furthermore, after selection in G418-containing cell culture medium, nearly all surviving colonies will stably express the gene of interest, thus decreasing the need to screen large numbers of colonies to find functional clones (Rees et al., 1996). Consequently, the expression of proteins which impair cell viability or genetic configurations imposing high counterselective constraints (such as controlled proliferation engineering, Fussenegger et al., 1997a) are expected to be selectable (Kaufman et al., 1991; Rees et al., 1996; Müller et al., 1997). Auto-selection also decreases the risk of genetic drift in bioprocesses (Rees et al., 1996).

Additionally, the CITE element contained in pQuattro harbours a reduction-in-function mutation which has been previously reported for the dicistronic expression vector pIRESneo (Rees et al., 1996; Clontech). The lower translation efficiency of the resistance cistron has been repeatedly shown to be compensated by the selection of chromosomal integration sites with overall high transcription activity (Kaufman, et al., 1991; Rees et al., 1996; Krömer et al., 1997).
Despite repetitive IRES elements, problems with genetic stability of the pQuattro vectors has never been observed neither during cloning or amplification in E.coli nor following transfection into mammalian cells (Fussenegger et al., 1997b and 1998). Taken together, the family of quattrocistronic mammalian expression vectors harbours the potential for coordinated multicistronic, auto-selective as well as regulatable expression of several transgenes in mammalian cells following a single engineering step. Owing to its modular set-up, pQuattro can be easily adapted to any state-ofthe-art expression technology currently evaluated for use in tissue engineering and gene therapy.

\section{Acknowledgement}

This work was supported by the Bundesamt für Bildung und Wissenschaft (BBW) within the Framework IV Biotechnology Program of the European Commission and the Swiss Priority Program in Biotechnology (SPP BioTech).

\section{References}

Berger J, Hauber J, Hauber G, Geiger R and Cullen BR (1988) Secreted alkaline phosphatase: A powerful new quantitative indicator of geneexpression in eukaryotic cells. Gene 66: 1-10.

Bernstein J, Sella O, Le SY and Elroy-Stein O (1997) PDGF2/C SIS mRNAleader contains a differentiation linked internal ribosomal entry site (DIRES). J Biol Chem 272: 9356-9362.

Crameri A, Whitehorn EA, Tate E and Stemmer WPC (1996) Improved green fluorescent protein by molecular evolution using DNA shuffling. Nature Biotechnology 14: 315-319.

Dirks W, Wirth M and Hauser H (1993) Dicistronic transcription units for gene expression in mammalian cells. Gene 128: 247249.

Dirks W, Schaper F, Kirchhoff S, Morelle C and Hauser H (1994) A multifunctional vector family for gene expression in mammlian cells. Gene 149: 387-388.

El-Deiry WW, Tokino T, Velculescu VE, Levy DB, Parsons R, Trent JM, Lin D, Mercer WE, Kinzler KW and Vogelstein B (1993) WAF1, a potential mediator of p53 tumor suppression. Cell 75: $817-825$.

Fussenegger M, Mazur X and Bailey JE (1997a) A novel cytostatic process enhances the productivity of Chinese hamster ovary cells. Biotechnol Bioeng 55: 927-939.

Fussenegger M, Moser S, Mazur X, Bailey JE (1997b) Autoregulated multicistronic expression vectors provide one-step cloning of regulatedproduct gene expression in mammalian cells. Biotech Prog 13: 733-740.

Fussenegger M, Bailey JE, Hauser H and Müller PP (1998a) Genetic optimizationof recombinant protein production by mammalian cells. TIBTECH (in press).

Fussenegger M, Schlatter S, Dätwyler D, Mazur X and Bailey JE (1998b) Controlled proliferation by multigene metabolic engineering enhancesthe productivity of Chinese hamster ovary cells. Nature Biotechnology 16: 468-472. 
Fussenegger M, Mazur X, Renner WA and Bailey JE (1998c) Higher productivity of growth-arrested Chinese hamster ovary (CHO) cells expressing the cyclin-dependent kinase inhibitor p27. Biotechnol Prog (in press).

Fussenegger M, Mazur X and Bailey JE (1998d) pTRIDENT, a novel vector family for tricistronic gene expression in mammalian cells. Biotech Bioeng 57: 1-10.

Kaufman RJ, Davis MV, Wasley LC and Micknick D 1991. Improved vectors for the stable expression of foreign genes in mammalian cells by use of the untranslated leader sequence from DMC virus. Nucl Acids Res 19: 4485-4490.

Krömer WJ, Carafoli E and Bailey JB (1997) Purification of the cardiacsarcoplasmic reticulum membrane protein phospholamban from recombinant E. coli. Eur J Biochem 248: 814-819.

Müller P, Kirchhoff S and Hauser, H (1998) Sustained expression in proliferation controlled BHK-21 cells. in New developments andapplications in animal cell technology, Merten OW, Perrin P and Griffiths JB (eds.), Kluwer Academic Publishers, Dordrecht, The Netherlands (in press).

Papoutsakis ET (1998) Express together and conquer. Nature Biotechnology 16: 416-417.

Rees S, Coote J, Stables J, Goodson S, Harris S and Lee MG (1996) Bicistronic vector for the creation of stable mammalian cell lines that predisposes all antibiotic-resistant cells to express recombinant protein. BioTechniques 20: 48-56. 